\title{
Solving delay differential equations by the five-point one-step block method using Neville's interpolation
}

\begin{abstract}
A five-point one-step block method based on the Newton backward divided difference formulae for the solution of first-order delay differential equations is derived. The proposed block method will approximate the solutions of initial value problems at five points simultaneously using variable step size. The approximation of the delay term is calculated using Neville's interpolation. The block method will be formulated in terms of linear multistep method, but the method is equivalent to one-step method. The order of the block method will be discussed. The P-stability and Q-stability regions of the block method using Neville's interpolation for the delay term are presented for a fixed step size. Numerical results are given to show the efficiency of the proposed method and compared with the existing method.
\end{abstract}

Keyword: Block method; Delay differential equations; Variable step size. 